\title{
A Framework for Dynamic Hedging under Convex Risk Measures
}

\author{
Antoine Toussaint* Ronnie Sircar ${ }^{\dagger}$
}

November 2008; revised August 2009

\begin{abstract}
We consider the problem of minimizing the risk of a financial position (hedging) in an incomplete market. It is well-known that the industry standard for risk measure, the Valueat-Risk, does not take into account the natural idea that risk should be minimized through diversification. This observation led to the recent theory of coherent and convex risk measures. But, as a theory on bounded financial positions, it is not ideally suited for the problem of hedging because simple strategies such as buy-hold strategies may not be bounded. Therefore, we propose as an alternative to use convex risk measures defined as functionals on $L^{2}$ (or by simple extension $L^{p}, p>1$ ). This framework is more suitable for optimal hedging with $L^{2}$ valued financial markets. A dual representation is given for this minimum risk or market adjusted risk when the risk measure is real-valued. In the general case, we introduce constrained hedging and prove that the market adjusted risk is still a $L^{2}$ convex risk measure and the existence of the optimal hedge. We illustrate the practical advantage in the shortfall risk measure by showing how minimizing risk in this framework can lead to a HJB equation and we give an example of computation in a stochastic volatility model with the shortfall risk measure
\end{abstract}

\section{Introduction}

We are interested in the problem of hedging in an incomplete market: an investor decides to buy a contract with a non-replicable payoff $X$ at time $T$ but has the opportunity to invest in a financial market to cover his risk. By convention, the hedge will be a short position obtained by trading with strategy $H$ and borrowing the discounted amount $\$ x$ from a riskless account. The final wealth for the investor is $X-\left(x+G_{T}(H)\right)$ where $G_{T}(H)$ is the wealth at time $T$ obtained through trading. The question of hedging is the choice of the best pair $(x, H)$ to minimize the total risk of the investor.

The standard for measuring risk in the industry, the VaR measure, has been criticized because it fails to take into account the economically justified notion of reduction of risk through diversification. Artzner et al. [1999] initiated an axiomatic approach to risk measures, namely coherent risk measures. Their axiom of homogeneity was relaxed later by Föllmer and Schied [2002] to define convex risk measures. However, these types of measures were defined only for bounded financial positions. This appears too restrictive first because many common derivatives like call options are not bounded. Also, in models not locally bounded, the only bounded investment is the zero investment. Therefore, it seems practical to extend this concept to convex risk measure defined on broader spaces. Certain spaces like $L^{p}$ spaces with $1<p<+\infty$ or Orlicz spaces $L^{\Phi}$ are natural candidates for different reasons.

Risk measures defined on $L^{\infty}$ are always finite as a simple consequence of the translation invariance and monotonicity. However it is easy to construct functionals on $L^{p}$ spaces, extension of convex risk measures on $L^{\infty}$, that can assume the value $+\infty$. A classical example is the entropic risk

\footnotetext{
*Mathematics Department, Stanford University, Building 380, Stanford, CA 94305; atoussai@stanford.edu

†ORFE Department, Princeton University, Sherrerd Hall, Princeton NJ 08544; sircar@princeton.edu. Work of both authors partially supported by NSF grant DMS-0456195.
} 
measure ent $(X)=\log \mathbb{E}[\exp (-X)]$ on $L^{1}$ for example. The question of $+\infty$ has been approached in two ways.

The first one requires the risk measure to stay finite and the right framework seems to be the very general extension to Orlicz hearts proposed by Cheridito and Li [2009] which requires convex measures to have a non-empty interior as stated in their Theorem 4.6 together with Lemma 4.1. This result has been generalized to general Frechet lattice spaces in Theorem 1 of Biagini and Frittelli [2009]. The other approach aims at enlarging the space while not guaranteeing the finiteness of the risk measure, for example in Ruszczynski and Shapiro [2004] and Rudloff [2006] for $L^{p}$ spaces, with Orlicz spaces in Biagini [2006] or Biagini et al. [2008]. In Kloppel and Schweizer [2006], the special case of convex risk measures defined through BSDEs gives an example of finitely valued measures defined on $L^{2}$.

Both approaches have advantages. Ensuring finiteness of the convex functional guarantees norm continuity and therefore a natural dual theory which was one of the central points in the development of convex risk measures on $L^{\infty}$. However, in the concern of hedging, general conditions of Orliczintegrability for the portfolio value do not appear to be explicit enough from a practical point of view. As an example, the entropic risk measure can be defined on a Orlicz heart where it takes finite values, but it is easy to see that in the case of a simple Black-Scholes model, the payoff of a short position on a call option does not have the appropriate integrability since $\mathbb{E}\left[\exp \left(\left(S_{T}-K\right)^{+}\right)\right]=+\infty$. However, it makes perfect sense to talk about hedging this position under this particular measure of risk since it can be checked easily that by investing in only buy-and-hold strategy, one can achieve a finite risk:

$$
\mathbb{E}\left[\exp \left(-\left\{S_{T}-\left(S_{T}-K\right)^{+}\right\}\right)\right]<+\infty .
$$

It seems more natural from a practical point of view, if one wants to consider common risk measures, such as the entropic one, to allow for the value $+\infty$. The major inconvenience of this point of view is that we lose a priori the natural duality theory since we need now to impose a condition of lower-semicontinuity which was automatic for finite convex functionals. An important recent contribution by Jouini et al. [2006] to the study of convex risk measures was the proof that convex risk measures on $L^{\infty}$ which are law invariant (as the entropic measure or any utility based risk measure) have automatically the Fatou property, and thus a clean dual representation in terms of probability measures. This powerful and fundamental result unfortunately doesn't extend to $\mathbb{R} \cup\{+\infty\}$-valued convex risk measures defined on $L^{p}$, as illustrated in Example 5.1 of Filipovic and Svindland [2008] when studying the problem of extending convex risk measures to larger spaces. The link between the Fatou property and pointwise (or order) lower-semicontinuity is investigated in detail in Biagini and Frittelli [2009]. It seems however for the sake of simplicity to require the condition of lower-semicontinuity to ensure the dual representation.

To be more precise, this condition of lower semicontinuity is the strongest norm continuity assumption one can make since continuity forces a fortiori the convex risk measures to be finite at least for $L^{p}$ spaces and certain Orlicz spaces. This result is a consequence of Lemma 2.6 in Filipovic and Svindland [2005].

So far, it seems necessary to impose a lower-semicontinuity condition. This allows a duality representation which is the common ground of all these frameworks. Therefore, it is the latter approach, with measures defined on a $L^{p}(\mathbb{P})$ space, $1 \leq p<\infty$, on which we will focus here. This duality question appears to us to be a crucial criteria for the choice of $L^{p}$. The choice of $L^{p}=L^{1}$ with dual $L^{q}=\left(L^{p}\right)^{*}=L^{\infty}$ is however not well adapted to market adjusted risk measures. Indeed, their dual representations would be written on bounded martingale measures - where we identify measures absolutely continuous with respect to $\mathbb{P}$ with their Radon-Nikodym derivatives. They is in general not possible in continuous time finance. For simplicity, we see in $L^{2}$ a natural candidate for a first approach but $L^{p}$ spaces would behave in a similar way. It fits the historical development of the theory of stochastic integration with semimartingales. Moreover, as we will see, a condition of existence of an optimal constrained hedging strategy relates to the closedness of the space of attainable claims $\left\{G_{T}(H)\right\}$, a question which appears naturally in the quadratic hedging theory (we refer to Schweizer [2001] for a survey on the subject). 
Therefore, our approach to hedging in incomplete markets will focus on the minimization

$$
X \mapsto \inf _{H} \rho\left(X-\left(x+G_{T}(H)\right)\right)
$$

where $\rho$ will be a convex risk measure defined on $L^{2}$. Minimal conditions will imposed on the set of trading strategies and the financial market for $G_{T}(H)$ to be square integrable and buy-hold strategies to be admissible.

\section{$2 \quad L^{2}$ Convex risk measures}

In this section, we define and study $L^{2}$ convex and coherent risk measures defined on the set of square integrable random variables.

\subsection{Definition and properties}

Let $(\Omega, \mathcal{F}, \mathbb{P})$ be a probability space. We write $L^{2}$ or $L^{2}(\mathbb{P})$ for $L^{2}(\Omega, \mathcal{F}, \mathbb{P})$ when no confusion is possible.

Definition 2.1. An $L^{2}$ convex risk measure is a proper functional $\rho: L^{2}(\mathbb{P}) \rightarrow \mathbb{R} \cup\{+\infty\}$ which satisfies the following properties: for all $X, Y,\left(X_{n}\right) \in L^{2}(\mathbb{P}), \alpha \in(0,1), m \in \mathbb{R}$,

(A1) Convexity: $\rho(\alpha X+(1-\alpha) Y) \leq \alpha \rho(X)+(1-\alpha) \rho(Y)$.

(A2) Monotonicity: $X \leq Y \mathbb{P}$-a.s $\Rightarrow \rho(X) \geq \rho(Y)$.

(A3) Translation Invariance: $\rho(X+m)=\rho(X)-m$.

(A4) Lower semicontinuity: $\left\|X_{n}-X\right\|_{L^{2}} \rightarrow 0$, then $\rho(X) \leq \liminf _{n} \rho\left(X_{n}\right)$.

If moreover, $\rho$ satisfies

(A5) Positive homogeneity: $\forall X \in L^{2}, t \geq 0, \rho(t X)=t \rho(X)$

then $\rho$ is called an $L^{2}$ coherent risk measure.

We see that there are two differences with the usual definition as seen in Föllmer and Schied [2002].

(1) We allow $\rho$ to take the value $+\infty$. Economically, a financial position $X$ such that $\rho(X)=+\infty$ is terrible: whatever the amount of cash we add, we cannot make this position acceptable, that is, find an amount of cash $m$ such that $\rho(X+m) \leq 0$. However, mathematically, real valued convex risk measures such as the $L^{2}$ shortfall are therefore easier to work with, since if $\rho$ is real valued (with value in $\mathbb{R}$ ) and satisfies $(A 1),(A 2),(A 3)$, then it automatically satisfies $(A 4)$.

(2) We introduce the lower semicontinuity as a part of the definition whereas, in the usual $L^{\infty}$ case, the translation invariance makes risk measures Lipschitz and therefore norm continuous. This property is a necessary condition to obtain any kind of dual representation and is preserved by inf-convolution under some compactness condition.

The property of translation invariance gives the risk measure the meaning of a cash requirement. The risk $\rho(X)$ is the minimum amount of cash to add to the position to obtain a zero risk, $\rho(X+$ $\rho(X))=0$. A position with a negative risk does not need any cash to make it riskless. We define the set of all financial positions with negative risk.

Definition 2.2. A financial position $X$ is said to be acceptable if $\rho(X) \leq 0$ and we define the acceptance set $\mathcal{A}_{\rho}$ of $\rho$ to be the the set of all acceptable positions:

$$
\mathcal{A}_{\rho}:=\{X \in \mathcal{X} \mid \rho(X) \leq 0\}
$$


The economic justification of the lower semicontinuity is less obvious a priori than the other three properties. However it can be checked that the lower semicontinuity is equivalent to the much more natural concept of continuity from above:

Definition 2.3. We say that $\rho$ is continuous from above: if $X_{n} \searrow X$ a.s., then

$$
\rho\left(X_{n}\right) \nearrow \rho(X) \text {. }
$$

Proposition 2.4. Let $\rho$ be an $L^{2}$ convex risk measure, then the following are equivalent

(i) $\rho$ is lower semicontinuous.

(ii) $\rho$ is continuous from above.

The proof was first established in the $L^{\infty}$ framework in Föllmer and Schied [2002] in Lemma 4.16. We reproduce it with the appropriate modifications to cover the $L^{2}$ case. A proof in a more general framework is given in Proposition 24 of Biagini and Frittelli [2009].

Proof. Suppose first that $(i)$ holds. Consider $X_{n} \searrow X$ a.s. By the monotonicity of $\rho,\left(\rho\left(X_{n}\right)\right)$ is increasing. Since $0 \leq X_{n}-X \leq X_{0}-X$, by the dominated convergence theorem, $\left(X_{n}\right)$ converges in $L^{2}$ to $X$. By the lower semicontinuity of $\rho, \rho(X) \leq \lim \inf \rho\left(X_{n}\right)$. Since $X_{n} \geq X$, we have also that $\rho\left(X_{n}\right) \leq \rho(X)$ so that $\lim \inf \rho\left(X_{n}\right) \leq \rho(X)$ and $\liminf \rho\left(X_{n}\right) \leq \lim \sup \rho\left(X_{n}\right) \leq \rho(X)$. So $\rho(X)=\liminf \rho\left(X_{n}\right)=\limsup \rho\left(X_{n}\right)=\lim \rho\left(X_{n}\right)$ and we get $\rho\left(X_{n}\right) \nearrow \rho(X)$ which is $(i i)$.

Suppose now that $(i)$ doesn't hold. There exists $\left(X_{n}\right)$ convergent to $X$ such that $\rho(X)>\lim \inf \rho\left(X_{n}\right)$. There exists a subsequence $\left(n_{k}\right)$ such that $\lim \rho\left(X_{n_{k}}\right)=\liminf \rho\left(X_{n}\right)$. Since $\left(X_{n_{k}}\right)$ converges also to $X$ in $L^{2}$, there exists a further subsequence $\left(k_{m}\right)$ such that $\left(X_{n_{k_{m}}}\right)$ converges pointwise to $X$. Define $Y_{m}=\sup _{i \geq m} X_{n_{k_{i}}}$, so that $\left(Y_{m}\right)$ decreases pointwise to $X$ almost surely. We also have by monotonicity that $\rho\left(Y_{m}\right) \leq \rho\left(X_{n_{k_{m}}}\right)$. We deduce that $\liminf \rho\left(Y_{m}\right) \leq \lim \inf \rho\left(X_{n_{k_{m}}}\right)=\liminf \rho\left(X_{n}\right)<\rho(X)$, which proves that (ii) doesn't hold.

We will see in two steps that lower semicontinuity is the strongest assumption that one can take which still allows for the value $+\infty$. A first result due to Ruszczynski and Shapiro [2004] in this framework is of great use when $\rho$ is real-valued, applied to the special $L^{2}$ framework.

Theorem 2.5. [Ruszczynski, Shapiro] Assume $\rho$ satisfies $(A 1),(A 2)$ on $L^{2}$, then $\rho$ is norm continuous and weakly lower semicontinuous on int $\operatorname{dom}(\rho)$. In particular, if $\rho$ satisfies also (A3) and is real valued, it is an $L^{2}$ convex risk measure.

For the proof of Theorem 2.5, we refer to Ruszczynski and Shapiro [2004]. Their proof shows also that real valued convex risk measures are subdifferentiable. We show now that if $\operatorname{dom}(\rho) \neq L^{2}(\mathbb{P})$, then int $\operatorname{dom}(\rho)=\emptyset$. We reproduce the proofs for their simplicity.

Proposition 2.6. If there exists $X$ such that $\rho(X)=+\infty$, then int $\operatorname{dom}(\rho)=\emptyset$.

In view of using Lemma 2.6 in Filipovic and Svindland [2005], we will need a small lemma first:

Lemma 2.7. Let $\rho$ be an $L^{p}$ convex risk measure, then

$$
\text { int } \operatorname{dom}(\rho)=\emptyset \Leftrightarrow \operatorname{int} \mathcal{A}_{\rho}=\emptyset \text {. }
$$

Proof of the proposition. Since $\rho$ is l.s.c. and $\operatorname{dom}(\rho) \neq L^{p}$, then by Lemma 2.6 in Filipovic and Svindland [2005], int $\mathcal{A}_{\rho}=\emptyset$ and by Lemma 2.7, int $\operatorname{dom}(\rho)=\emptyset$. This result also appears as a remark in Filipovic and Svindland [2008].

Proof of the lemma. Since $\mathcal{A}_{\rho} \subset \operatorname{dom}(\rho)$, ' $\Rightarrow$ ' is immediate. To prove the other direction, assume that int $\operatorname{dom}(\rho) \neq \emptyset$, and pick $x_{0}$ in this interior. There exists $\delta>0$ such that $B\left(x_{0}, \delta\right) \subset \operatorname{dom}(\rho)$. By a classical result of convex analysis, $\rho$ is continuous on $B\left(x_{0}, \delta\right)$ and so for $\epsilon>0$, there exists $0<$ $\delta^{\prime} \leq \delta$ such that for all $x \in B\left(x_{0}, \delta^{\prime}\right),\left|\rho(x)-\rho\left(x_{0}\right)\right| \leq \epsilon$ so that $\rho(x)-\rho\left(x_{0}\right)-\epsilon=\rho\left(x+\rho\left(x_{0}\right)+\epsilon\right) \leq 0$ which means that $B\left(x_{0}+\rho\left(x_{0}\right)-\epsilon, \delta^{\prime}\right) \subset \mathcal{A}_{\rho}$ and therefore int $\mathcal{A}_{\rho} \neq \emptyset$. 


\subsection{Dual representation of $L^{2}$ convex risk measures}

Standard results of convex analysis allow to derive a dual representation of $L^{2}$ convex risk measures. For proofs, we refer for example to Filipovic and Svindland [2005]. We denote by $\mathcal{P}_{a}^{2}$ the probability measures absolutely continuous to $\mathbb{P}$ with square integrable Radon-Nikodym derivatives:

$$
\mathcal{P}_{a}^{2}:=\left\{\mathbb{Q} \ll \mathbb{P} \mid \mathbb{E}\left(\frac{d \mathbb{Q}}{d \mathbb{P}}\right)^{2}<+\infty\right\},
$$

and $\mathcal{P}_{e}^{2}$ the subset of $\mathcal{P}_{a}^{2}$ of measures which are also equivalent to $\mathbb{P}$.

Theorem 2.8. Let $\rho$ be an $L^{2}$ convex risk measure. Then $\rho$ admits the dual representation:

$$
\rho(X)=\sup _{\mathbb{Q} \in \mathcal{P}_{a}^{2}}\left\{\mathbb{E}_{\mathbb{Q}}[-X]-\alpha(\mathbb{Q})\right\}
$$

for a functional $\alpha: \mathcal{P}_{a}^{2} \mapsto \mathbb{R} \cup\{+\infty\}$ defined in terms of the acceptance set of $\rho$ as

$$
\alpha(\mathbb{Q}):=\sup _{X \in \mathcal{A}_{\rho}} \mathbb{E}_{\mathbb{Q}}[-X] .
$$

If $\rho$ is real-valued, we can replace the sup in the dual representation by a max. If $\operatorname{dom}(\alpha) \cap \mathcal{P}_{e}^{2} \neq \emptyset$, then we have

$$
\rho(X)=\sup _{\mathbb{Q} \in \mathcal{P}_{e}^{2}}\left\{\mathbb{E}_{\mathbb{Q}}[-X]-\alpha(\mathbb{Q})\right\} .
$$

When the risk measure is also coherent, we have a nicer dual representation.

Theorem 2.9. Let $\rho$ be an $L^{2}$ coherent risk measure. Then $\rho$ admits the dual representation:

$$
\rho(X):=\sup _{\mathbb{Q} \in \mathcal{Q}} \mathbb{E}_{\mathbb{Q}}[-X]
$$

where $\mathcal{Q}$ is a closed convex subset of $\mathcal{P}_{a}^{2}$. Moreover, in terms of its acceptance set $\mathcal{A}_{\rho}$ :

$$
\mathcal{Q}=\mathcal{A}_{\rho}^{\circ}:=\left\{\mathbb{Q} \in \mathcal{P}_{a}^{2} \mid \mathbb{E}_{\mathbb{Q}}[-X] \leq 0, \text { for all } X \in \mathcal{A}_{\rho}\right\} .
$$

If $\rho$ is also real-valued, then $\mathcal{Q}$ is $L^{2}$ bounded and weakly compact and we can replace the sup in the dual representation by a max.

Note that the sup cannot be replaced by a max in general for a risk measure possibly taking the value $+\infty$ even when $\rho(X)$ itself is finite as illustrated in Example 1. We give two well-known examples of risk measures, namely the entropic risk measure and the $L^{2}$ expected shortfall, which are $L^{2}$ convex risk measures, and we give their dual representations.

\subsection{Examples: entropic and $L^{2}$ shortfall risk measures}

Both examples belong to the natural class of utility based shortfall risk and are therefore law invariant. Giesecke and Weber [2008] analyse the qualitative behavior of these utility based measures of risk and justify their use for looking at large losses when they become much more suitable than the Value at Risk (which fails in general to be convex).

Definition 2.10. For every $X \in L^{2}(\mathbb{P})$, we define the entropic risk measure $\rho$ by

$$
\rho(X)=\log \mathbb{E} \exp (-X) .
$$

Proposition 2.11. The entropic risk measure is an $L^{2}$ convex risk measure. In particular, it is lower semicontinuous. It is continuous from above. Continuous from below is however only assured on its domain. 
Proof. These follow from simple application of Fatou's lemma and the dominated convergence theorem.

Proposition 2.12. The entropic convex measure admits the following dual representation

$$
\rho(X)=\sup _{\mathbb{Q} \in \mathcal{P}_{a}^{2}}\left\{\mathbb{E}_{\mathbb{Q}}[-X]-H(\mathbb{Q} \mid \mathbb{P})\right\}
$$

Using the example of the entropic risk measure, we show here a case where the sup in the dual representation is not attained although $\rho(X)<+\infty$.

Example 1. Consider $\Omega=\{1,2, \ldots\}, \mathcal{F}=2^{\Omega}, \mathbb{P}$ defined by $\mathbb{P}\{\omega=n\}=p_{n}=a / n^{3}, n \geq 1$ where $a$ is chosen for normalization. Now, consider the random variable $X$ defined by $X(n)=-\log (b n)=: x_{n}$ for $b>0$ to be chosen later. $X$ is in $L^{2}(\mathbb{P})$ for all $b$ since $\sum|\log (n b)| / n^{3}$ is convergent. We look at the following equivalent problem:

$$
-\rho(X)=\inf _{\mathbb{Q} \in \mathcal{P}_{a}^{2}}\left\{\mathbb{E}_{\mathbb{Q}}[X]+H(\mathbb{Q} \mid \mathbb{P})\right\}=: \bar{A} .
$$

and the extended problem (by dropping the square integrability of the Radon-Nikodym derivative of $\mathbb{Q})$ :

$$
\underline{A}:=\inf _{\mathbb{Q} \in \mathcal{P}_{a}}\left\{\mathbb{E}_{\mathbb{Q}}[X]+H(\mathbb{Q} \mid \mathbb{P})\right\}
$$

We can prove that $\bar{A}=\underline{A} \in \mathbb{R}$ and that there exists a unique minimizer reaching $\bar{A}$ which does not belong to $\mathcal{P}_{a}^{2}$ and leave the details to the reader. Therefore our claim is proved.

We can define a real-valued risk measure, the $L^{2}$ shortfall measure of risk, via its acceptance set, that is, financial positions which we find acceptable in the sense that their expected losses are smaller than a certain threshold. This was studied in some detail under the name Utility Based Risk measure by Giesecke and Weber [2008].

Definition 2.13. For $x_{0}>0$, we define the set of financial positions with losses bounded in $L^{2}$ :

$$
\mathcal{A}:=\left\{X \in \mathcal{X} \mid \mathbb{E}\left[\left(X^{-}\right)^{2}\right] \leq x_{0}\right\},
$$

and the $L^{2}$-shortfall at level $x_{0}$ is defined for every $X \in L^{2}$ by

$$
\begin{aligned}
\rho(X) & :=\inf \{m \in \mathbb{R} \mid m+X \in \mathcal{A}\} \\
& =\inf \left\{m \in \mathbb{R} \mid \mathbb{E}\left[(m+X)^{-}\right]^{2} \leq x_{0}\right\} .
\end{aligned}
$$

It is straightforward to check that the shortfall risk measure satisfies the following.

Proposition 2.14. The $L^{2}$ shortfall is a real valued $L^{2}$ convex risk measure and its set of acceptable position is $\mathcal{A}$. For every $X \in L^{2}, \rho(X)$ is the unique solution $m \in \mathbb{R}$ of the equation

$$
\mathbb{E}\left[(m+X)^{-}\right]^{2}=x_{0} .
$$

Proposition 2.15. The $L^{2}$-shortfall convex measure $\rho$ admits the following dual representation

$$
\rho(X)=\max _{\mathbb{Q} \in \mathcal{P}_{a}^{2}}\left\{\mathbb{E}_{\mathbb{Q}}[-X]-\sqrt{2 x_{0}}\left\|\frac{d \mathbb{Q}}{d \mathbb{P}}\right\|_{2}\right\} \text {. }
$$

Proof. We proved that $\operatorname{dom}(\rho)=L^{2}(\mathbb{P})$ so $\rho$ is a lower semicontinuous convex risk measure and therefore admits a dual representation with penalty function

$$
\alpha(\mathbb{Q})=\sup _{X \in \mathcal{A}}\left\{\mathbb{E}_{\mathbb{Q}}[-X]\right\}
$$

The rest of the proof is identical of the one of Theorem 4.61 by Föllmer and Schied [2002] except there is no need for localization due to the larger class of random variables $L^{2}$.

We will see that the problem of optimal hedging can be viewed as a particular case of infconvolution of a risk measure. The next subsection studies the properties of this operator in an $L^{2}$ framework. 


\subsection{Inf-convolution of risk measures}

The inf-convolution of convex risk measures was introduced in Barrieu and Karoui [2004] and Barrieu and Karoui [2005] in the $L^{\infty}$ framework where we could use monotonicity pointwise convergence results so that the lower-semicontinuity was obtained in generality. In an $L^{2}$ framework, since we cannot reduce norm convergence to pointwise convergence, the lower semicontinuity of the infconvolution is not automatic.

Definition 2.16. Let $\rho$ be an $L^{2}$ convex risk measure and $\phi$ a functional on $L^{2}(\mathbb{P})$. We define the inf-convolution of $\rho$ and $\phi$ as

$$
\rho \square \phi(X):=\inf _{Y \in L^{2}(\mathbb{P})}\{\rho(X-Y)+\phi(Y)\}=\inf _{Y \in L^{2}(\mathbb{P})}\{\rho(Y)+\phi(X-Y)\}
$$

The inf-convolution of an $L^{2}$ convex risk measure with a functional is in some cases again an $L^{2}$ convex risk measure. As in Barrieu and Karoui [2004] and Barrieu and Karoui [2005], it is easy to check that for any $\phi, \rho \square \phi$ has the monotonicity and cash translation invariance property. For $\rho \square \phi$ to be an $L^{2}$ convex risk measure, we need therefore to impose conditions for $\rho \square \phi$ to be $\mathbb{R} \cup\{+\infty\}$ valued, i.e. $\rho \square \phi$ to be proper, convex and lower semicontinuous. Unfortunately, so far, we cannot derive a general theorem, so we divide into two cases, when $\rho$ is real-valued and when it isn't, where we impose more conditions on $\phi$.

Proposition 2.17. Suppose that $\rho$ is a real valued $L^{2}$ convex risk measure. If $\phi$ is convex, proper, such that $\rho \square \phi$ is proper, then the inf-convolution $\rho \square \phi$ is also a real valued $L^{2}$ convex risk measure and admits the dual representation in the sense of convex risk measures with penalty function

$$
\alpha_{\rho \square \phi}(\mathbb{Q})=\alpha_{\rho}(\mathbb{Q})+\alpha_{\phi}(\mathbb{Q})
$$

where

$$
\alpha_{\phi}(\mathbb{Q}):=\sup _{X \in L^{2}(\mathbb{P})}\left\{\mathbb{E}_{\mathbb{Q}}[X]-\phi(X)\right\} .
$$

Proof. We refer to Toussaint [2007].

If we drop the assumption on the continuity of $\rho$, then we need first to ensure that $\phi$ is lower semicontinuous as well. The inf-convolution of two convex lower semicontinuous functionals is not trivially lower semicontinuous. In fact, a necessary and sufficient condition for $\rho \square \phi$ to be lower semicontinuous is epi $(\rho)+\operatorname{epi}(\phi)$ to be closed. But since the sum of convex closed subsets may not be closed, the lower semicontinuity of the inf-convolution is not automatic. However, if the domain of $\phi$ is weakly compact, then we can obtain the following result:

Proposition 2.18. Suppose that $\rho$ is an $L^{2}$ convex risk measure. If $\phi$ is convex, proper and lower semicontinuous with dom $(\phi)$ weakly compact and $\operatorname{dom}(\rho) \cap \operatorname{dom}(\phi) \neq \emptyset$, then the inf-convolution $\rho \square \phi$ is also an $L^{2}$ convex risk measure and admits the dual representation in the sense of convex risk measures with penalty function

$$
\alpha_{\rho \square \phi}(\mathbb{Q})=\alpha_{\rho}(\mathbb{Q})+\alpha_{\phi}(\mathbb{Q}) .
$$

Proof. Since we can write,

$$
\rho \square \phi(X)=\inf _{Y \in L^{2}(\mathbb{P})}\{\rho(X-Y)+\phi(Y)\}=\inf _{Y \in \operatorname{dom}(\phi)}\{\rho(X-Y)+\phi(Y)\}
$$

and since $Y \mapsto \rho(X-Y)+\phi(Y)$ is weakly lower semicontinuous (since lower semicontinuous and convex), then either $\rho \square \phi(X)=+\infty$ or $\rho \square \phi(X)$ is finite since we minimize a lower semicontinuous functional on a weakly compact set. And since $\operatorname{dom}(\rho) \cap \operatorname{dom}(\phi) \neq \emptyset, \rho \square \phi$ is proper. As in the previous proof, the convexity, the monotonicity and the translation invariance properties are satisfied. We need to check the lower semicontinuity. We want to check that, for all $r \in \mathbb{R}$,

$$
S(r):=\left\{X \in L^{2}(\mathbb{P}) \mid \rho \square \phi(X) \leq r\right\}
$$


is closed. Consider a sequence $\left(X_{n}\right)$ in $S(r)$ convergent to some $X$. For every $\epsilon>0$, there exists $Y_{n} \in \operatorname{dom}(\phi)$ such that

$$
\rho\left(X_{n}-Y_{n}\right)+\phi\left(Y_{n}\right) \leq r+\epsilon
$$

Since $\left(Y_{n}\right)$ is a sequence in a weakly compact set, there exists a weakly convergent subsequence $\left(Y_{n_{k}}\right)$ converging to some $Y$ in $\operatorname{dom}(\phi)$. Since $X_{n_{k}} \rightarrow X$ also, $X_{n_{k}}-Y_{n_{k}}$ converges weakly to $X-Y$, and since $\rho$ and $\phi$ are weakly lower semicontinuous,

$$
\begin{aligned}
\rho(X-Y)+\phi(Y) & \leq \liminf \rho\left(X_{n_{k}}-Y_{n_{k}}\right)+\liminf \phi\left(Y_{n_{k}}\right) \\
& \leq \liminf \rho\left(X_{n_{k}}-Y_{n_{k}}\right)+\phi\left(Y_{n_{k}}\right) \leq r+\epsilon .
\end{aligned}
$$

Since this holds for every $\epsilon$, we deduce that $X \in S(r)$ which completes the claim.

In view of applying this result to optimal hedging, we investigate the case where $\phi$ is an indicator function (in the sense of convex analysis) of a convex set in $L^{2}(\mathbb{P})$ where the proof is a simple corollary of the previous result.

Proposition 2.19. Let $C$ be a non-empty convex, closed subset of $L^{2}$ and define the functional $\phi$ on $L^{2}$ to be the indicator function of $C$, in the sense of convex analysis. That is, for all $X \in L^{2}(\mathbb{P})$

$$
\phi(X):=\delta_{C}(X):= \begin{cases}0, & \text { if } x \in C \\ +\infty & \text { otherwise. }\end{cases}
$$

Then $\phi$ is a proper convex, lower semicontinuous functional. If $C$ is bounded, then $\operatorname{dom}(\phi)=C$ is weakly compact. If $C$ is a symmetric cone, i.e. $\mathbb{R}_{+} C:=\{\lambda c \mid c \in C, \lambda \geq 0\}=C$ and $-C:=\{-c \mid c \in C\}=C$ then its penalty function as defined in Proposition 2.17 is:

$$
\alpha_{\phi}(\mathbb{Q})=\delta_{C^{\perp}}(d \mathbb{Q} / d \mathbb{P})
$$

where $C^{\perp}$ is the orthogonal of $C$.

With these results of stability of lower semicontinuity, we can introduce the concept of a $C$ constrained $L^{2}$ convex risk measure, where $C$ is a subset of $L^{2}(\mathbb{P})$.

Definition 2.20. Suppose that $\rho$ is an $L^{2}$ convex risk measure, we define the $C$-constrained $L^{2}$ convex measure $\rho^{C}$ by:

$$
\rho^{C}(X):=\inf _{Y \in C} \rho(X-Y)
$$

This can be written as a special case of an inf-convolution of $\rho$ :

$$
\rho^{C}(X)=\inf _{Y \in L^{2}(\mathbb{P})}\left\{\rho(X-Y)+\delta_{C}(Y)\right\}=\rho \square \delta_{C}(X) .
$$

Proposition 2.21. Suppose $C$ is a symmetric convex cone and $\rho$ is a real valued $L^{2}$ convex risk measure with penalty function $\alpha$ such that $\rho \square \delta_{C}$ is proper, then $\rho^{C}=\rho \square \delta_{C}$ is again an $L^{2}$ convex risk measure and has the following dual representation

$$
\rho^{C}(X):=\sup _{\mathbb{Q} \in \mathcal{P}_{a}^{2} \cap \bar{C}^{\perp}}\left\{\mathbb{E}_{\mathbb{Q}}[-X]-\alpha(\mathbb{Q})\right\} .
$$

Proof. Since $\rho$ is continuous, $\rho^{C}=\rho^{\bar{C}}$ and the proof is a direct consequence of Propositions 2.17 and 2.19 .

In the case where $\rho$ is only lower semicontinuous, we need to consider only bounded sets $C$, so the inf-convolution defines again an $L^{2}$ convex risk measure.

Proposition 2.22. Suppose $C$ is a bounded closed convex set and $\rho$ is an $L^{2}$ convex risk, then $\rho^{C}=\rho \square \delta_{C}$ is again an $L^{2}$ convex risk measure.

Proof. This is a direct consequence of Propositions 2.18 and 2.19.

In the next section, we define a financial market with mathematical properties matching the framework of $L^{2}$ convex risk measures. In particular, we insist that in common models simple buy-and-hold or buy-sell strategies lead to square integrable terminal wealth. 


\section{Dynamic hedging in incomplete markets}

\subsection{Arbitrage restrictions on the financial market}

In general, investment strategies in the financial markets are given by predictable processes satisfying some integrability condition sufficient to rule out inconveniences such as doubling or suicide strategies. In the seminal paper of Harrison and Pliska [1981] and in most general literature on arbitrage mathematics, see for example Delbaen and Schachermayer [2006], the convention is to consider strategies in $L(S)$ such that the portfolio value is bounded from below by a constant independent of time, economically justified as the line of credit of the investor. However, we can imagine models where this restriction is problematic, namely models where the price process is not locally bounded: discrete time models in the first place but also continuously traded models with unbounded unpredictable jumps where no short strategy satisfies the lower bound. Generalizations to a random lower bound have been proposed and investigated in particular in Biagini and Fritelli [2005]. We choose a different approach and do not require any bound on the portfolio value. However, we require a stronger integrability of strategies to match our $L^{2}$ framework: our maximal set of trading strategies will be $L^{2}(S)$ instead of the more general $L(S)$. By definition, $G_{T}(H) \in L^{2}(\mathbb{P})$. We consider also stronger integrability condition on the semimartingale to ensure that a large class of strategies produces a terminal wealth in $L^{2}(\mathbb{P})$. As we would like this class of strategies to contain simple bounded strategies, we will consider $\mathcal{H}^{2}$ semimartingales as defined in Protter [2005]. For the sake of completeness $X \in \mathcal{H}^{2}$ if $X=M+A$ where $\left(M_{t}\right)_{t \in[0, T]}$ is a squared integrable martingale and $A$ a predictable process of finite variation such that $\int_{0}^{T}\left|d A_{t}\right|$ is square integrable. We have

$$
\left(H_{1}\right): \quad S \in \mathcal{H}^{2}(\mathbb{P}) \text { and } \Theta:=L^{2}(S) .
$$

We remark that we do not require uniform boundedness from below for the value of the portfolio $G_{t}(H)$, where

$$
G_{t}(H):=\int_{0}^{t} H_{u} d S_{u}:=(H \cdot S)_{t} .
$$

If $\left(H_{1}\right)$ is satisfied then all uniformly bounded strategies are admissible. In the section on bounded trading strategies, we will use the assumption that the financial market is $L^{2}$ closed, i.e.

$$
\left(H_{2}\right): \quad G_{T}(\Theta):=\left\{\int_{0}^{T} H_{t} d S_{t}, H \in \Theta\right\} \text { is closed for the } L^{2} \text { norm. }
$$

Necessary and sufficient conditions for the case $S$ continuous are given in Delbaen et al. [1997]. See also Schweizer [2001] for a survey.

The investor's measure of risk is given by an $L^{2}$ convex risk measure $\rho$ as defined in Definition 2.1: compared to the $L^{\infty}$ framework, $\rho$ takes values in $\mathbb{R} \cup\{+\infty\}$ and is required to be lowersemicontinuous. Every $L^{2}$ convex risk measure admits a dual representation with a penalty function $\alpha$ defined on the set $\mathcal{P}_{a}^{2}$.

Often, we identify $\mathbb{Q}$ and $d \mathbb{Q} / d \mathbb{P}$. We denote by $\mathcal{M}_{a}$ (resp. $\mathcal{M}_{e}$ ) the set of absolutely continuous (resp. equivalent) local martingale measures for $S$, and we define:

$$
\mathcal{M}_{a}^{2}:=\left\{\mathbb{Q} \in \mathcal{P}_{a}^{2} \cap \mathcal{M}_{a}\right\} ; \quad, \mathcal{M}_{e}^{2}:=\left\{\mathbb{Q} \in \mathcal{P}_{a}^{2} \cap \mathcal{M}_{e}\right\} .
$$

The condition of no arbitrage we will use here is the following:

$$
\left(H_{3}^{\prime}\right): \quad \mathcal{M}_{e}^{2}:=\left\{\mathbb{Q} \in \mathcal{P}_{a}^{2} \cap \mathcal{M}_{e}\right\} \neq \emptyset,
$$

and it can be proved that $\left(H_{3}^{\prime}\right)$ implies the classical no arbitrage condition (NA). We define the stronger assumption:

$$
\left(H_{3}\right): \quad \mathcal{M}_{e, f}^{2}:=\left\{\mathbb{Q} \in M_{e}^{2} \mid \alpha(\mathbb{Q})<+\infty\right\} \neq \emptyset
$$


This condition is a simple sufficient condition for the market adjusted risk measure to be proper which is economically relevant as we will explain after Theorem 3.4.

What is important here is the following characterization of martingale measures as the orthogonal space of trading strategies:

Theorem 3.1. Let $S$ be in $\mathcal{H}^{2}$. Then the following are equivalent:

(i) $\mathbb{Q} \in \mathcal{M}_{a}^{2}(\mathbb{P})$,

(ii) $\mathbb{Q} \in \mathcal{P}_{a}^{2}(\mathbb{P})$ and $\mathbb{E}_{\mathbb{Q}}\left[(H \cdot S)_{T}\right]=0$ for all $H \in L^{2}(S)$.

The same equivalence holds with equivalent probability measures instead of only absolutely continuous ones.

The proof and details on notations are given in Toussaint [2007].

\subsection{Market adjusted risk measures}

The problem of optimal hedging with $\$ x$ corresponds to the objective of minimizing the risk of $X-\left(x+G_{T}(H)\right)$ over all admissible trading strategies $H \in \Theta$ :

$$
\inf _{H \in \Theta} \rho\left(X-\left(x+G_{T}(H)\right)\right) .
$$

We define for all $X$ :

$$
\rho^{G_{T}(\Theta)}(X):=\inf _{H \in \Theta} \rho\left(X-G_{T}(H)\right) .
$$

We notice that $\rho^{G_{T}(\Theta)}$, the market adjusted risk, is the inf-convolution of $\rho$ with the indicator function of a convex set in $L^{2}$. A goal of this paper is to extend the results in $L^{\infty}$ of Barrieu and Karoui [2004] and Barrieu and Karoui [2005] when possible and in particular obtain a dual representation for $\rho^{G_{T}(\Theta)}$, that is to ask the question if $\rho^{G_{T}(\Theta)}$ is again an $L^{2}$ convex risk measure: this is a structural question. In some cases, this is not an immediate extension: in $L^{\infty}$, the dual representation is equivalent to a point-wise monotonic lower-semicontinuity. The monotonicity of $\rho$ makes it easier to obtain the same property for the inf-convolution of $\rho$. However, in $L^{2}$, the dual representation is equivalent to a norm lower-semicontinuity and thus the same conclusion does not hold. When $\rho$ is finitely-valued, it is easy to extend this standard result to our framework.

Theorem 3.2. Suppose $\left(H_{1}\right)$ and $\left(H_{3}\right)$ hold true. Let $\rho$ be a real valued $L^{2}$ convex risk measure, then $\rho^{G_{T}(\Theta)}$ is again a real-valued $L^{2}$ convex risk measure and it has the following dual representation:

$$
\rho^{G_{T}(\Theta)}(X)=\sup _{\mathbb{Q} \in \mathcal{M}_{e, f}^{2}}\left\{\mathbb{E}_{\mathbb{Q}}[-X]-\alpha(\mathbb{Q})\right\} .
$$

The proof is given in the appendix.

For risk measures which can take the value $+\infty$, we have seen that the optimal hedge does not define immediately another well-behaved $L^{2}$ convex risk measure because of the uncertainty of the lower semicontinuity of the inf-convolution. We investigate therefore the economically relevant case of an investor who would only be allowed to invest with bounded strategies. In this case, we will see that the question of the existence of an optimal hedge depends on some closedness property of the set of final trading wealth.

We can suppose an investor only allows for the hedging strategies where the allocation in the asset are limited by a set $\mathcal{K}$. For example, he could restrict his hedging portfolio to only have a positive fraction of the asset, in that case $\mathcal{K}=[0,1]$. A set of constraints $\mathcal{K} \subset \mathbb{R}^{+}$forbids short selling in general.

For a trading constraints set $\mathcal{K}$, we define the set $\Theta_{\mathcal{K}}$ of trading strategies lying in a convex compact set $\mathcal{K}$, i.e.

$$
\Theta_{\mathcal{K}}=\left\{H \in L^{2}(S) \mid H(t, \omega) \in \mathcal{K} \text { for all }(t, \omega)\right\} .
$$


A weaker condition is needed as detailed in Toussaint [2007]. Here, the problem of constrained optimal hedging is:

$$
\rho^{G_{T}\left(\Theta_{\mathcal{K}}\right)}:=\inf _{H \in \Theta_{\mathcal{K}}} \rho\left(X-G_{T}(H)\right) .
$$

We have these two results, whose proof are given in the appendix:

Theorem 3.3. Suppose that $\left(H_{1}\right),\left(H_{2}\right)$ and $\left(H_{3}\right)$ hold true. Let $\rho$ be an $L^{2}$ convex risk measure, then $\rho^{G_{T}\left(\Theta_{\mathcal{K}}\right)}$ is an $L^{2}$ convex risk measure.

Theorem 3.4. Suppose that $\left(H_{1}\right),\left(H_{2}\right)$ and $\left(H_{3}\right)$ hold true. Let $\rho$ be an $L^{2}$ convex risk measure, then there exists an optimal hedge in $\Theta_{\mathcal{K}}$.

The proofs are given in the appendix.

We remark that the properness of $\rho^{G_{T}(\Theta)}$, namely that it is not possible to obtain an arbitrary low risk through hedging is economically sane and should be true in any model.If it were not true for a certain payoff $X$, for any amount of cash $x$, we could find a hedging strategy $H$ such that $\rho\left(X-(H \cdot S)_{T}-x\right) \leq 0$. In other words, we could take away any amount of money from our portfolio and still find a hedging strategy that makes the total position acceptable in term of risk.

One advantage of the dual representation is that it is equivalent to a stochastic control problem where the control determines the set of martingale measures. However, to use dynamic programming methods, one often asks for strong integrability on the control, much stronger generally that what is necessary to define in our case a martingale measure which could be the reason of a gap between the primal problem and the HJB solution, a classical example being the Merton problem and the difficulty in incomplete markets to prove that the HJB solution is indeed equal to the primal problem. We will provide in the last section a case study of the $L^{2}$ shortfall risk within a stochastic volatility model, and a condition for which the set of controls can be taken to be square integrable.

\section{Shortfall Risk in a stochastic volatility model}

In the Merton problem with a classical complete Black-Scholes model, it is well known that the optimal terminal wealth (Brownian Motion plus a drift, calculated from the Hamilton Jacobi Bellman equation) is not in $L^{\infty}$, but it is in $L^{2}$, so the formulation on $L^{\infty}$ is not satisfactory (i.e. not that the HJB solution is suboptimal) but a $L^{2}$ framework is more robust in that view.

We consider the Brownian-based stochastic volatility model. The model consists of two correlated assets when only one can be traded and an option is written on possibly the two assets.

$$
\left\{\begin{array}{l}
d S_{t}=\mu\left(Y_{t}\right) S_{t} d t+\sigma\left(Y_{t}\right) S_{t} d W_{t}^{(0)}, \quad S_{0}=s \\
d Y_{t}=b\left(Y_{t}\right) d t+a\left(Y_{t}\right)\left(c d W_{t}^{(0)}+\bar{c} d W_{t}^{(1)}\right), \quad Y_{0}=y,
\end{array}\right.
$$

where $\left(W^{(0)},\left(W^{(1)}\right)\right.$ are independent Brownian motions, $c \in(0,1), \bar{c}=\sqrt{1-c^{2}}$. The filtration $\left(\mathcal{F}_{t}\right)_{t \in[0, T]}$ is the augmented filtration generated by $\left(W^{(0)},\left(W^{(1)}\right)\right.$ on $[0, T]$. We assume that $\mu$ and $\sigma$ are continuous and bounded functions, with $\sigma(\cdot) \geq \underline{\sigma}>0$. We assume also that $b$ and $a$ are globally Lipschitz. These conditions guarantee that both $Y$ and $S$ exist as strong solution of the system of SDEs. We write (31) in integral form:

$$
S_{t}=S_{0}+\int_{0}^{t} \mu\left(Y_{u}\right) S_{u} d u+\int_{0}^{t} \sigma\left(Y_{u}\right) S_{u} d W_{u}=A_{t}+M_{t},
$$

where $A_{t}:=S_{0}+\int_{0}^{t} \mu\left(Y_{u}\right) S_{u} d u$ and $M_{t}:=\int_{0}^{t} \sigma\left(Y_{u}\right) S_{u} d W_{u}$ and we can prove that $S$ is a $\mathcal{H}^{2}$ semimartingale and its unique decomposition is given as above by noticing that the coefficients are bounded and that $Y$ is continuous and $\mu(\cdot)$ and $\sigma(\cdot)$ are continuous. In this case, in formal 
notations, $d A_{t}=\mu\left(Y_{t}\right) S_{t} d t$ where $\mu(Y)$.$S . is indeed predictable as it is adapted and continuous; and$ $d M_{t}=\sigma S_{t} d W_{t}$, so

$$
d\langle M, M\rangle_{t}=\sigma\left(Y_{t}\right)^{2} S_{t}^{2} d t
$$

and we can write

$$
d A_{t}=\frac{\mu\left(Y_{t}\right)}{\sigma\left(Y_{t}\right)^{2} S_{t}} d\langle M, M\rangle_{t}=\lambda_{t} d\langle M, M\rangle_{t}
$$

where $\lambda_{t}:=\frac{\mu\left(Y_{t}\right)}{\sigma\left(Y_{t}\right)^{2} S_{t}}$. We remark that since $\lambda$ is adapted and continuous, it is therefore predictable and we check that

$$
K_{t}=\int_{0}^{t} \lambda_{u}^{2} d\langle M, M\rangle_{u}=\int_{0}^{t} \frac{\mu\left(Y_{u}\right)^{2}}{\sigma\left(Y_{u}\right)^{4} S_{u}^{2}} \sigma\left(Y_{u}\right)^{2} S_{u}^{2} d u \leq \frac{\bar{\mu}^{2}}{{\underline{\sigma^{2}}}^{2}} t<+\infty
$$

where $\bar{\mu}$ and $\underline{\sigma}$ are respectively the upper and lower bound of $\mu(\cdot)$ and $\sigma(\cdot)$. The uniform bound on $K$ is a strong condition as seen in Delbaen et al. [1997] for $\left(H_{2}\right)$ to be satisfied.

We would like to consider European-style options on $S, Y$, i.e. contracts where the payoff can be written $X:=h\left(S_{T}, Y_{T}\right) \in L^{2}(\mathbb{P})$ where $h$ is some positive function, and try to solve numerically the optimal hedging problem under the $L^{2}$ shortfall risk. By Proposition 2.14, the $L^{2}$ shortfall is a real-valued $L^{2}$ convex risk measure.

We prove that assumption $\left(H_{3}\right)$ holds true, i.e. that $\mathcal{M}_{e, f}^{2}$ is not empty. By the assumption of boundedness on the coefficient, we can check that the Novikov's condition holds for $\frac{\mu\left(Y_{.}\right)}{\sigma\left(Y_{Y}\right)} \cdot W^{(0)}$ so $\mathcal{E}_{T}\left(\frac{\mu\left(Y_{.}\right)}{\sigma\left(Y_{.}\right)} \cdot W^{(0)}\right)$ is the Radon-Nikodym derivative of an element $\mathbb{Q}^{0}$ of $\mathcal{M}_{e, f}^{2}$. This element is usually called the minimal martingale measure and we will write $\lambda^{0}=\frac{\mu\left(Y_{.}\right)}{\sigma\left(Y_{.}\right)}$. Therefore, we know from Theorem 3.2 that

$$
\inf _{H \in \Theta} \rho\left(X-(H \cdot S)_{T}\right)=\sup _{\mathbb{Q} \in \mathcal{M}_{e, f}^{2}}\left\{\mathbb{E}_{\mathbb{Q}}[-X]-\sqrt{2 x_{0}}\left\|\frac{d \mathbb{Q}}{d \mathbb{P}}\right\|_{2}\right\}
$$

But by Proposition B.2.1 in Musiela and Rutkowski [1998], for every measure $\mathbb{Q}$ equivalent to $\mathbb{P}$, there exists an adapted process $\lambda$ such that $d \mathbb{Q} / d \mathbb{P}=\mathcal{E}_{T}(\lambda \cdot W)$, where $W=\left(W^{(0)}, W^{(1)}\right)$. Since the first coordinate of $\lambda$ has to be $\mu(Y$.) $/ \sigma(Y$.), the only freedom is on the second coordinate, which we write $\lambda$ as well. Define then the following set of adapted processes:

$$
\begin{array}{r}
\Lambda:=\left\{\lambda \text { adapted, } \int_{0}^{T} \lambda_{t}^{2} d t<+\infty, \text { a.s, } \mathbb{E}\left[\mathcal{E}_{T}\left(\lambda^{0} \cdot W^{(0)}+\lambda \cdot W^{(1)}\right)\right]=1,\right. \\
\left.\mathbb{E}\left[\mathcal{E}_{T}\left(\lambda^{0} \cdot W^{(0)}+\lambda \cdot W^{(1)}\right)^{2}\right]<+\infty\right\} .
\end{array}
$$

This complicated set characterizes completely the set of martingale measures whose RadonNikodym derivatives have finite second moments. For each element $\lambda$ of $\Lambda$, we define $\mathbb{Q}^{\lambda}$ by

$$
\frac{d \mathbb{Q}^{\lambda}}{d \mathbb{P}}=\mathcal{E}_{T}\left(\lambda^{0} \cdot W^{(0)}+\lambda \cdot W^{(1)}\right)
$$

We remark that by definition, $0 \in \Lambda$ so our previous notation for the minimal measure $\mathbb{Q}^{0}$ is consistent. Through this characterization, we can write the problem of risk minimization as

$$
\inf _{H \in \Theta} \rho\left(X+(H \cdot S)_{T}\right)=\sup _{\lambda \in \Lambda}\left\{\mathbb{E}_{\mathbb{Q}^{\lambda}}[-X]-\sqrt{2 x_{0}}\left\|\mathbb{Q}^{\lambda}\right\|_{2}\right\}=: \sup _{\lambda \in \Lambda} U\left(X, \mathbb{Q}^{\lambda}, x_{0}\right) .
$$

This dual is not expressed as the expectation under $\mathbb{P}$ of a random quantity because of the term $\mathbb{E}\left[\left(d \mathbb{Q}^{\lambda} / d \mathbb{P}\right)^{2}\right]^{2}=\left(\mathbb{E}_{\mathbb{Q}^{\lambda}}\left[d \mathbb{Q}^{\lambda} / d \mathbb{P}\right]\right)^{2}$. We follow Ilhan et al. [2009] to obtain from there a problem that can be solved with dynamic programming methods. 
Recall that the quantity we are looking for in the optimal hedging problem is

$$
u\left(x_{0}\right):=\sup _{\lambda \in \Lambda} U\left(X, \mathbb{Q}^{\lambda}, x_{0}\right),
$$

where $U\left(X, \mathbb{Q}, x_{0}\right)=\mathbb{E}_{\mathbb{Q}}[-X]-\sqrt{2 x_{0}}\|d \mathbb{P} / d \mathbb{Q}\|_{2}$. We remark that, as a function of $x_{0}, U$ is convex and continuous. Therefore, we can apply Fenchel's theorem, fixing $\mathbb{Q}$ and $X$ (which we don't write):

$$
U\left(\mathbb{Q}, x_{0}\right)=\sup _{z>0}\left(\hat{U}(\mathbb{Q}, z)-z x_{0}\right) \text { where } \hat{U}(z, \mathbb{Q}):=\inf _{x_{0}>0}\left(U\left(\mathbb{Q}, x_{0}\right)+z x_{0}\right) .
$$

But on the other side,

$$
u\left(x_{0}\right)=\sup _{\lambda \in \Lambda} \sup _{z>0}\left(\hat{U}(\mathbb{Q}, z)-z x_{0}\right)=\sup _{z>0} \sup _{\lambda \in \Lambda}\left(\hat{U}(\mathbb{Q}, z)-z x_{0}\right),
$$

and $\hat{U}$ has the nicer expression

$$
\hat{U}(\mathbb{Q}, z)=\mathbb{E}_{\mathbb{Q}}[-X]-\frac{1}{2 z} \mathbb{E}\left[\left(\frac{d \mathbb{Q}}{d \mathbb{P}}\right)^{2}\right]=\mathbb{E}\left[-Z_{T} X-\frac{1}{2 z} Z_{T}^{2}\right]
$$

where $Z_{T}$ is the terminal value of the Radon-Nikodym derivatives $\left((d \mathbb{Q} / d \mathbb{P})_{\mathcal{F}_{t}}\right)$. Therefore the computation of the optimal risk becomes

$$
\sup _{\lambda \in \Lambda} \mathbb{E}\left[-Z_{T}^{\lambda} X-\frac{1}{2 z}\left(Z_{T}^{\lambda}\right)^{2}\right]
$$

As in Ilhan et al. [2009], we notice that this computation can approached through dynamic programming and has an associated Hamilton-Jacobi-Bellman equation as long as $X$ is a European claim in a Markovian framework. However to use this approach, an additional condition of integrability for $\lambda$ is needed. In particular, the specification of $\int_{0}^{T} \lambda_{t}^{2} d t<+\infty$, a.s is not suitable, and we would like instead to deal with processes such that $\mathbb{E}\left[\int_{0}^{T} \lambda_{t}^{2} d t\right]<+\infty$. We define

$$
\begin{array}{r}
\Gamma:=\left\{\lambda \text { adapted, } \mathbb{E}\left[\int_{0}^{T} \lambda_{t}^{2} d t\right]<+\infty, \mathbb{E}\left[\mathcal{E}_{T}\left(\lambda^{0} \cdot W^{(0)}+\lambda \cdot W^{(1)}\right)\right]=1,\right. \\
\left.\mathbb{E}\left[\mathcal{E}_{T}\left(\lambda^{0} \cdot W^{(0)}+\lambda \cdot W^{(1)}\right)^{2}\right]<+\infty\right\} .
\end{array}
$$

The next proposition gives a condition under which optimization over $\Gamma$ has the same value as optimizing over $\Lambda$.

We define the set of processes $\Lambda^{+} \subset \Lambda$ by

$$
\begin{array}{r}
\Lambda^{+}:=\left\{\lambda \text { adapted, } \int_{0}^{T} \lambda_{t}^{2} d t<+\infty, \text { a.s, } \mathbb{E}\left[\mathcal{E}_{T}\left(\lambda^{0} \cdot W^{(0)}+\lambda \cdot W^{(1)}\right)\right]=1,\right. \\
\left.\mathbb{E}\left[\mathcal{E}_{T}\left(\lambda^{0} \cdot W^{(0)}+\lambda \cdot W^{(1)}\right)^{(2+\delta)}\right]<+\infty, \text { for some } \delta>0\right\} .
\end{array}
$$

and adopt the following assumption:

$$
\left(H^{*}\right): \sup _{\lambda \in \Lambda} U\left(X, \mathbb{Q}^{\lambda}, x_{0}\right)=\sup _{\lambda \in \Lambda^{+}} U\left(X, \mathbb{Q}^{\lambda}, x_{0}\right) .
$$

In other words, the optimization can be achieved over probability measures whose Radon-Nikodym derivatives have slightly better integrability than square integrability. The meaning of this hypothesis and validity goes beyond the scope of this short introduction.

We prove the following result:

Proposition 4.1. Suppose $\left(H^{*}\right)$ holds, then

$$
\underline{u}:=\sup _{\lambda \in \Gamma} U\left(X, \mathbb{Q}^{\lambda}, x_{0}\right)=\sup _{\lambda \in \Lambda} U\left(X, \mathbb{Q}^{\lambda}, x_{0}\right)=: \bar{u} .
$$

We refer to the Appendix for the proof. The problem for $\bar{u}$ is studied by numerical PDE methods in Ilhan et al. [2009]. By viewing the dynamic hedging problem in the context of $L^{2}$ convex risk measures, it is now possible to reconcile the results of dynamic programming computations with the abstract theory. 


\section{Conclusion}

We developed a framework for risk measures that is well-suited to dynamic hedging in a financial market. It imposes reasonable integrability conditions, namely that the final wealth of trading strategies be square integrable. $L^{2}$ convex risk measures are natural within this framework and the additional axiom of lower semicontinuity drops when the risk measure is real-valued, so they are close to the original $L^{\infty}$ functional developed in the literature. The integrability assumption on the financial market made it possible to write simple no arbitrage conditions even when dropping the more typical assumption that the portfolio's wealth should be bounded from below. We are able to characterize the problem of risk minimization in the language of the inf-convolution of convex risk measures, which is used to write a dual representation for the market adjusted risk measure when the convex risk measure is real-valued. When the convex risk measure can attain the value $+\infty$, the lower semicontinuity of the market adjusted risk measure doesn't seem to be automatic. It is true however when only hedging portfolios with bounded strategy are considered. In that case, the strong assumption of closedness of the space of attainable claims is useful to obtain the existence of an optimal hedge since it enables the weak compactness of the usable strategies. Although this core property has been completely solved for continuous assets in Delbaen et al. [1997], it is still to be studied in more general cases.

\section{Appendix}

\subsection{Proof of Theorem 3.2}

First, we remark that the translation invariance property for convex risk measures makes the question of initial investment for the trading strategies irrelevant. Indeed,

$$
\inf _{H \in \Theta} \rho\left(X-\left(x+\int_{0}^{T} H_{t} d S_{t}\right)\right)=x+\inf _{H \in \Theta} \rho\left(X-\int_{0}^{T} H_{t} d S_{t}\right)
$$

and therefore we will consider only optimization with respect to investment in the financial market

with $\$ 0$ initial capital. Note now that the optimal hedging problem can be expressed in terms of inf-convolution of $\rho$ :

$$
\inf _{H \in \Theta} \rho\left(X-G_{T}(H)\right)=\rho^{G_{T}(\Theta)}(X) .
$$

We check easily that the main assumptions of Proposition 2.21 are already verified and we only need to verify that $\rho \square \delta_{G_{T}(\Theta)}$ is proper. But since we supposed the assumption $\left(H_{3}\right)$ to be satisfied, there exists $\mathbb{Q}^{*} \in \mathcal{M}_{e, f}^{2}(\mathbb{P})$. Therefore,

$$
\begin{aligned}
\rho^{G_{T}(\Theta)}(X) & =\inf _{H \in \Theta} \sup _{\mathbb{Q} \in \mathcal{P}_{e}^{2}}\left\{\mathbb{E}_{\mathbb{Q}}[-X]+\mathbb{E}_{\mathbb{Q}}\left[(H \cdot S)_{T}\right]-\alpha(\mathbb{Q})\right\} \\
& \geq \sup _{\mathbb{Q} \in \mathcal{P}_{e}^{2}} \inf _{H \in \Theta}\left\{\mathbb{E}_{\mathbb{Q}}[-X]+\mathbb{E}_{\mathbb{Q}}\left[(H \cdot S)_{T}\right]-\alpha(\mathbb{Q})\right\} \\
& =\sup _{\mathbb{Q} \in \mathcal{M}_{e, f}^{2}}\left\{\mathbb{E}_{\mathbb{Q}}[-X]-\alpha(\mathbb{Q})\right\} \geq \mathbb{E}_{\mathbb{Q}^{*}}[-X]-\alpha\left(\mathbb{Q}^{*}\right)>-\infty
\end{aligned}
$$

where the last equality comes from the fact that if $\mathbb{Q}$ is not a martingale measure, then $\inf _{H \in \Theta} \mathbb{E}\left[(H \cdot S)_{T}\right]=-\infty$ since $\Theta$ is a symmetric cone. Now we note that $G_{T}(\Theta)$ is trivially a convex symmetric cone and it follows by Proposition 2.21,

$$
\rho^{G_{T}(\Theta)}(X)=\sup _{\mathbb{Q} \in \mathcal{P}_{a}^{2} \cap \overline{G_{T}(\Theta)}}\left\{\mathbb{E}_{\mathbb{Q}}[-X]-\alpha(\mathbb{Q})\right\} .
$$

If $\mathbb{Q} \in \mathcal{P}_{a}^{2} \cap \overline{G_{T}(\Theta)}{ }^{\perp}$, then $\mathbb{E}_{\mathbb{Q}}[f]=0$ for all $f \in \overline{G_{T}(\Theta)}$, which is by Theorem 3.1 equivalent to $\mathbb{Q} \in \mathcal{M}_{a}^{2}(\mathbb{P})$. Therefore

$$
\rho^{G_{T}(\Theta)}(X)=\sup _{\mathbb{Q} \in \mathcal{M}_{a}^{2}}\left\{\mathbb{E}_{\mathbb{Q}}[-X]-\alpha(\mathbb{Q})\right\}
$$


and we go from $\mathcal{M}_{a}^{2}$ to $\mathcal{M}_{e, f}^{2}$, which is non-empty by $\left(H_{3}\right)$, by denseness.

\subsection{Proof of Theorems 3.3 and 3.4}

The investor is only allowed the set $\Theta_{\mathcal{K}}$ of $\mathcal{K}$-constrained strategies where $\mathcal{K}$ is a convex compact subset of $\mathbb{R}$. We recall that since $\left(H_{2}\right)$ holds, it can be proved that $\mathcal{G}_{T}\left(\Theta_{\mathcal{K}}\right)$ is weakly compact. The result that $\rho^{G_{T}\left(\Theta_{\mathcal{K}}\right)}$ is an $L^{2}$ convex risk measure follows directly from Proposition 2.22 since $G_{T}\left(\Theta_{\mathcal{K}}\right)$ is a closed convex bounded set. Since an admissible strategy $H^{*} \in \Theta_{\mathcal{K}}$ is said to be $\mathcal{K}$-optimal if

$$
\rho\left(G-\left(H^{*} \cdot S\right)_{T}\right)=\inf _{H \in \Theta_{\mathcal{K}}} \rho\left(G-(H \cdot S)_{T}\right),
$$

the existence of an optimal hedge is equivalent to the existence of a minimizer $Y^{*} \in G_{T}\left(\Theta_{\mathcal{K}}\right)$ for

$$
\inf _{Y \in G_{T}\left(\Theta_{\mathcal{K}}\right)} \rho(G-Y) .
$$

Since $\rho$ is an $L^{2}$ convex risk measure, it is in particular convex and lower semicontinuous and is therefore also weakly lower semicontinuous. Since $G_{T}\left(\Theta_{\mathcal{K}}\right)$ is weakly compact and since a lower semicontinuous function attains its minimum on a compact, $\inf _{Y \in G_{T}\left(\Theta_{\mathcal{K}}\right)} \rho(G-Y)$ is attained by some $Y^{*}=\left(H^{*} \cdot S\right)_{T} \in G_{T}\left(\Theta_{\mathcal{K}}\right)$.

\subsection{Proof of Proposition 4.1}

The inequality $\underline{u} \leq \bar{u}$ is obvious. By assumption, for any $\epsilon>0$, there exists $\lambda \in \Lambda^{+}$such that $U\left(X, \mathbb{Q}^{\lambda}, x_{0}\right) \geq \bar{u}-\epsilon$. By definition of the stochastic integral, there exists a localizing sequence of stopping times $\left(\tau_{n}\right)$ such that $\mathbb{E} \int_{0}^{T}\left(\lambda_{t}^{\tau_{n}}\right)^{2} d t<+\infty, \int_{0}^{T}\left(\lambda_{t}^{\tau_{n}}\right)^{2} d t \stackrel{\mathbb{P}}{\rightarrow} \int_{0}^{T} \lambda_{t}^{2} d t$ and $\int_{0}^{T} \lambda_{t}^{\tau_{n}} d W_{t}^{(1)} \stackrel{\mathbb{P}}{\rightarrow}$ $\int_{0}^{T} \lambda_{t} d W_{t}^{(1)}$ where $\lambda_{t}^{\tau_{n}}=\lambda_{t} 1\left(t \leq \tau_{n}\right)$. Since $x \mapsto \exp (x)$ is continuous, we also have

$$
\mathcal{E}_{T}\left(\lambda^{0} \cdot W^{(0)}+\lambda_{t}^{\tau_{n}} \cdot W^{(1)}\right) \stackrel{\mathbb{P}}{\rightarrow} \mathcal{E}_{T}\left(\lambda^{0} \cdot W^{(0)}+\lambda \cdot W^{(1)}\right) .
$$

We want to show that $\lambda^{\tau_{n}}$ is in $\Gamma$ and that the limit in the previous expression can be taken in the $L^{2}$ sense as well.

We remark that since $\left\langle W^{(0)}, W^{(1)}\right\rangle=0$, we can decompose the Doleans exponential: $\mathcal{E}_{t}\left(\lambda_{0}\right.$. $\left.W^{(0)}+\lambda \cdot W^{(0)}\right)=\mathcal{E}_{t}\left(\lambda_{0} \cdot W^{(0)}\right) \mathcal{E}_{t}\left(\lambda \cdot W^{(1)}\right)$. But by the hypothesis on the coefficient and using Novikov's condition, $\mathcal{E}_{T}\left(\lambda^{0} \cdot W^{(0)}\right)$ defines a probability measure $\mathbb{Q}^{0}$ under which $W^{(0)}-\int_{0}^{\cdot} \lambda_{t}^{0} d t$ and $W^{(1)}$ are Brownian motions. We can write $\mathbb{E}\left[\mathcal{E}_{T}\left(\lambda^{0} \cdot W^{(0)}+\lambda \cdot W^{(1)}\right)\right]=\mathbb{E}^{0}\left[\mathcal{E}_{T}\left(\lambda \cdot W^{(1)}\right)\right]=1$ which proves that $\left(\mathcal{E}_{t}\left(\lambda \cdot W^{(1)}\right)\right)_{0 \leq t \leq T}$ is a $\mathbb{Q}^{0}$-martingale (it was a local martingale since $W^{(1)}$ is a $\mathbb{Q}^{0}$ Brownian motion), so by optimal stopping theorem for $\tau_{n} \leq T$,

$$
\mathbb{E}\left[\mathcal{E}_{T}\left(\lambda^{0} \cdot W^{(0)}+\lambda_{t}^{\tau_{n}} \cdot W^{(1)}\right)\right]=\mathbb{E}^{0}\left[\mathcal{E}_{T}\left(\lambda^{\tau_{n}} \cdot W^{(1)}\right)\right]=\mathbb{E}^{0}\left[\mathcal{E}_{\tau_{n}}\left(\lambda \cdot W^{(1)}\right)\right]=1 .
$$

We prove an auxiliary result: $\left(\mathcal{E}_{t}\left(\lambda \cdot W^{(1)}\right)\right)_{t \leq T}$ is a uniformly integrable $\mathbb{P}$ martingale (and not merely a $\mathbb{P}$ local martingale). We prove first that $\left(\mathcal{E}_{t}\left(\lambda \cdot W^{(1)}\right)\right)_{t \leq T}$ is of class $(D)$, i.e. that

$$
\left\{\mathcal{E}_{\tau}\left(\lambda \cdot W^{(1)}\right), \tau \leq T \text {, stopping time }\right\} \text { is uniformly integrable. }
$$

We will prove that there exists a constant $C$ such that $\mathbb{E}\left[\mathcal{E}_{\tau}\left(\lambda \cdot W^{(1)}\right)^{1+\delta / 2}\right] \leq C$ for any stopping time $\tau \leq T$ for some positive $\delta$. By assumption, there exists $\delta>0$ such that $\mathbb{E}\left[\mathcal{E}_{T}\left(\lambda^{0} \cdot W^{(0)}+\lambda\right.\right.$. $\left.\left.W^{(1)}\right)^{(2+\delta)}\right]<+\infty$. Now we write

$$
\begin{aligned}
\mathbb{E}\left[\mathcal{E}_{\tau}\left(\lambda \cdot W^{(1)}\right)^{1+\delta / 2}\right]^{2} & =\mathbb{E}\left[\left(\mathcal{E}_{\tau}\left(\lambda_{0} \cdot W^{(0)}\right)\right)^{-(1+\delta / 2)} \mathcal{E}_{\tau}\left(\lambda_{0} \cdot W^{(0)}\right)^{1+\delta / 2} \mathcal{E}_{\tau}\left(\lambda \cdot W^{(1)}\right)^{1+\delta / 2}\right]^{2} \\
& \leq \mathbb{E}\left[\left(\mathcal{E}_{\tau}\left(\lambda_{0} \cdot W^{(0)}\right)\right)^{-(2+\delta)}\right] \mathbb{E}\left[\left(\mathcal{E}_{\tau}\left(\lambda_{0} \cdot W^{(0)}\right) \mathcal{E}_{\tau}\left(\lambda \cdot W^{(1)}\right)\right)^{2+\delta}\right] \\
& \leq \mathbb{E}\left[\left(\mathcal{E}_{\tau}\left(\lambda_{0} \cdot W^{(0)}\right)\right)^{-(2+\delta)}\right] \mathbb{E}\left[\left(\mathcal{E}_{\tau}\left(\lambda_{0} \cdot W^{(0)}+\lambda \cdot W^{(1)}\right)\right)^{2+\delta}\right] \\
& \leq \mathbb{E}\left[\left(\mathcal{E}_{T}\left(\lambda_{0} \cdot W^{(0)}\right)\right)^{-(2+\delta)}\right] \mathbb{E}\left[\left(\mathcal{E}_{T}\left(\lambda_{0} \cdot W^{(0)}\right) \mathcal{E}_{T}\left(\lambda \cdot W^{(1)}\right)\right)^{2+\delta}\right] \leq C^{\prime} \cdot C
\end{aligned}
$$


where we used that $\left.\left(\mathcal{E} .\left(\lambda_{0} \cdot W^{(0)}\right)\right)^{-(2+\delta)}\right)$ and $\left.\left(\mathcal{E} .\left(\lambda_{0} \cdot W^{(0)}+\lambda \cdot W^{(1)}\right)\right)^{2+\delta}\right)$ are submartingales as the image of martingales by convex functions (on $\mathbb{R}^{+}$) and the optional sampling theorem where the existence of $C^{\prime}$ is given by Lemma 5.1. Now it is well known that a local martingale of class (D) is a uniformly integrable martingale. We proved that under $\left(H^{*}\right)$, both $\mathcal{E} .\left(\lambda^{0} \cdot W^{(0)}\right)$ and $\mathcal{E} .\left(\lambda \cdot W^{(1)}\right)$ are continuous uniformly integrable $\mathbb{P}$ martingales.

Fix $p=\frac{2+\delta}{2+\delta / 2}$ and note that we can pick $\delta$ such that $p \in(1,2)$. Define $q$ such that $p^{-1}+q^{-1}=1$. We apply Holder's inequality:

$$
\begin{aligned}
& \mathbb{E}\left[\mathcal{E}_{T}\left(\lambda^{0} \cdot W^{(0)}+\lambda^{\tau_{n}} \cdot W^{(1)}\right)^{2+\delta / 2}\right]=\mathbb{E}\left[\mathcal{E}_{T}\left(\lambda^{0} \cdot W^{(0)}\right)^{2+\delta / 2} \mathcal{E}_{\tau_{n}}\left(\lambda \cdot W^{(1)}\right)^{2+\delta / 2}\right] \\
& =\mathbb{E}\left[\left(\mathcal{E}_{\tau_{n}}\left(\lambda^{0} \cdot W^{(0)}\right)^{-(2+\delta / 2)} \mathcal{E}_{T}\left(\lambda^{0} \cdot W^{(0)}\right)^{2+\delta / 2}\right)\left(\mathcal{E}_{\tau_{n}}\left(\lambda^{0} \cdot W^{(0)}\right)^{2+\delta / 2} \mathcal{E}_{\tau_{n}}\left(\lambda \cdot W^{(1)}\right)^{2+\delta / 2}\right)\right] \\
& =\mathbb{E}\left[\left(\mathcal{E}_{\tau_{n}}\left(\lambda^{0} \cdot W^{(0)}\right)^{-(2+\delta / 2)} \mathcal{E}_{T}\left(\lambda^{0} \cdot W^{(0)}\right)^{2+\delta / 2}\right)\left(\mathcal{E}_{\tau_{n}}\left(\lambda^{0} \cdot W^{(0)}+\lambda \cdot W^{(1)}\right)\right)^{2+\delta / 2}\right] \\
& \leq A_{n}^{1 / q} B_{n}^{1 / p}
\end{aligned}
$$

where

$$
A_{n}:=\mathbb{E}\left[\left(\mathcal{E}_{\tau_{n}}\left(\lambda^{0} \cdot W^{(0)}\right)^{-q(2+\delta / 2)} \mathcal{E}_{T}\left(\lambda^{0} \cdot W^{(0)}\right)^{q(2+\delta / 2)}\right)\right]
$$

and

$$
B_{n}:=\mathbb{E}\left[\left(\mathcal{E}_{\tau_{n}}\left(\lambda^{0} \cdot W^{(0)}+\lambda \cdot W^{(1)}\right)\right)^{p(2+\delta / 2)}\right]=\mathbb{E}\left[\left(\mathcal{E}_{\tau_{n}}\left(\lambda^{0} \cdot W^{(0)}+\lambda \cdot W^{(1)}\right)\right)^{2+\delta}\right] .
$$

We show that these two terms are uniformly bounded in $n$.

We start with $\left(B_{n}\right)$ : since $\mathcal{E}$. $\left(\lambda^{0} \cdot W^{(0)}+\lambda \cdot W^{(1)}\right)$ is a martingale, $\mathcal{E} .\left(\lambda^{0} \cdot W^{(0)}+\lambda \cdot W^{(1)}\right)^{2+\delta}$ is a submartingale and by optional sampling theorem on $\tau_{n} \leq T$,

$$
B_{n} \leq \mathbb{E}\left[\left(\mathcal{E}_{T}\left(\lambda^{0} \cdot W^{(0)}+\lambda \cdot W^{(1)}\right)\right)^{2+\delta}\right]<+\infty
$$

by hypothesis. For $\left(A_{n}\right)$, we can use for example Cauchy-Schwartz inequality so that

$$
\begin{aligned}
A_{n}^{2} & \leq \mathbb{E}\left[\mathcal{E}_{\tau_{n}}\left(\lambda^{0} \cdot W^{(0)}\right)^{-2 q(2+\delta / 2)}\right] \mathbb{E}\left[\mathcal{E}_{T}\left(\lambda^{0} \cdot W^{(0)}\right)^{2 q(2+\delta / 2)}\right] \\
& \leq \mathbb{E}\left[\mathcal{E}_{T}\left(\lambda^{0} \cdot W^{(0)}\right)^{-2 q(2+\delta / 2)}\right] \cdot C<+\infty
\end{aligned}
$$

where we used again a submartingale inequality $\left(x \mapsto x^{-2 q(2+\delta / 2)}\right.$ is convex on $\mathbb{R}^{+}$for our $\left.q\right)$ and Lemma 5.1 twice.

In other word, this proved that $\mathcal{E}_{T}\left(\lambda^{0} \cdot W^{(0)}+\lambda^{\tau_{n}} \cdot W^{(1)}\right)$ is bounded in $L^{2+\delta / 2}$ and therefore $\mathcal{E}_{T}\left(\lambda^{0} \cdot W^{(0)}+\lambda^{\tau_{n}} \cdot W^{(1)}\right)^{2}$ is uniformly integrable. But since we had before that $\mathcal{E}_{T}\left(\lambda^{0} \cdot W^{(0)}+\right.$ $\left.\lambda^{\tau_{n}} \cdot W^{(1)}\right)$ converged to $\mathcal{E}_{T}\left(\lambda^{0} \cdot W^{(0)}+\lambda \cdot W^{(1)}\right)$ in probability, both results combined prove that $\mathcal{E}_{T}\left(\lambda^{0} \cdot W^{(0)}+\lambda^{\tau_{n}} \cdot W^{(1)}\right)$ converges to $\mathcal{E}_{T}\left(\lambda^{0} \cdot W^{(0)}+\lambda \cdot W^{(1)}\right)$ in $L^{2}$ as well.

We can finally proceed to our conclusion, since $U\left(X, \cdot, x_{0}\right)$ is continuous for the $L^{2}$ norm, we can find a $\lambda_{n} \in \Gamma$ such that $U\left(X, \mathbb{Q}^{\lambda^{n}}, x_{0}\right) \geq \bar{u}-2 \epsilon$ which proves that $\underline{u} \geq \bar{u}$ and therefore the equality holds.

Lemma 5.1. Suppose that $z$ is an adapted bounded process in the previous Brownian motion filtration. Then,

$$
\mathbb{E}\left[\exp \left((z \cdot W)_{T}\right)\right]<\infty .
$$

In particular, the Doleans exponential $\mathcal{E}_{T}(z \cdot W)$ has finite moments at any power. 
Proof. Since there exists $C$ such that $|z| \leq C$, then $\langle z \cdot W\rangle_{T}=\int_{0}^{T} z_{t}^{2} d t \leq C^{2} T$ and therefore $\mathbb{E}\left[\exp \left(\langle z \cdot W\rangle_{T}\right)\right]<+\infty$ and by Novikov's condition, $\mathcal{E}_{T}(z \cdot W)$ is a martingale. In particular,

$$
\mathbb{E}\left[\exp \left((z \cdot W)_{T}\right)\right]=\mathbb{E}\left[\mathcal{E}_{T}(z \cdot W) \exp \left(\frac{1}{2} \int_{0}^{T} z_{t}^{2} d t\right)\right] \leq \mathbb{E}\left[\mathcal{E}_{T}(z \cdot W)\right] \exp \left(1 / 2 C^{2} T\right)<+\infty .
$$

Take any power $p \in \mathbb{R}$,

$$
\mathbb{E}\left[\mathcal{E}_{T}(z \cdot W)^{p}\right]=\mathbb{E}\left[\exp \left((p z \cdot W)_{T}\right) \exp \left(-\frac{p}{2} \int_{0}^{T} z_{t}^{2} d t\right)\right] \leq \mathbb{E}\left[\exp (p z \cdot W)_{T}\right]
$$

and we use the previous result with the bounded process $\left(p z_{t}\right)$.

\section{References}

P. Artzner, F. Delbaen, J.M. Eber, and D Heath. Coherent measures of risk. Math. Finance, 9(3): 203-228, 1999.

P. Barrieu and N. El Karoui. Optimal derivatives design under dynamic risk measures. Article in Mathematics of Finance, Contemporary Mathematics (A.M.S. Proceedings), 13-26, 2004.

P. Barrieu and N. El Karoui. Inf-convolution of risk measures and optimal risk transfer. Finance and Stochastics, 9:269-298, 2005.

S. Biagini. An Orlicz spaces duality for utility maximization in incomplete markets. Progress in Probability, 2006.

S. Biagini and M. Fritelli. Utility maximization in incomplete markets for unbounded processes. Finance and Stochastics, 9:493-517, 2005.

S. Biagini and M. Frittelli. On the extension of the namioka-klee theorem and on the fatou property for risk measures. Optimality and risk: modern trends in mathematical finance, 2009.

S. Biagini, M. Fritelli, and M. Grasselli. Indifference price with general semimartingales. Submitted, 2008.

P. Cheridito and T. Li. Risk measures on Orlicz hearts. Mathematical Finance, 19(2):189-214, 2009.

F. Delbaen and W. Schachermayer. The mathematics of arbitrage. Springer Finance, 2006.

F. Delbaen, P. Monat, W. Schachermayer, M. Schweizer, and C. Stricker. Weighted norm inequalities and closedness of a space of stochastic integrals. Finance and Stochastics, 1:181-227, 1997.

D. Filipovic and G. Svindland. Convex risk measures on $L^{p}$. Working Paper, 2005.

D. Filipovic and G. Svindland. Convex risk measures beyond bounded risks, or the canonical model space for law-invariant convex risk measures is $L^{1}$. 2008. A shorter version to appear in Mathematical Finance.

H. Föllmer and A. Schied. Stochastic Finance, An Introduction in Discrete Time. Walter de Gruyter, 2002.

K. Giesecke and S. Weber. Measuring the risk of large losses. Journal of Investment Management, $6(4): 1-15,2008$.

M. Harrison and S. Pliska. Martingales and stochastic integrals in the theory of continuous trading. Stochastic Processes and Their Applications, 11:215-260, 1981.

A. Ilhan, M. Jonsson, and R. Sircar. Optimal static-dynamic hedge for exotic options under convex risk measures. Stochastic Processes and their Applications, 2009. In press. 
E. Jouini, W. Schachermayer, and N. Touzi. Law invariant risk measures have the fatou property. Advances in Mathematical Economics, 9:49-72, 2006.

S. Kloppel and Martin Schweizer. Dynamic utility indifference valuation via convex risk measures. Mathematical Finance, 209, 2006.

M. Musiela and P. Rutkowski. Martingale Methods in Financial Modelling. Springer, 1998.

P. Protter. Stochastic integration and differential equations, second edition. Springer-Verlag, 2005.

B. Rudloff. Hedging in incomplete markets and testing compound hypotheses via convex duality. Thesis Dissertation, 2006.

A. Ruszczynski and A. Shapiro. Optimization of convex risk functions. Risk and Insurance, 2004.

M. Schweizer. A guided tour through quadratic hedging approaches. Option Pricing, Interest Rates and Risk Management, Cambridge University Press, E. Jouini, J. Cvitanic, M. Musiela (eds.), pages 538-574, 2001.

A. Toussaint. Hedging with $L^{2}$ convex risk measures. Dissertation, Princeton University, 2007. 